\title{
Gradhiva
}

GRADHIV

Revue d'anthropologie et d'histoire des arts

32 | 2021

Livres sorciers

\section{La part chinoise de Wifredo Lam. Perspectives historiques et esthétiques}

Wifredo Lam's Chinese Side. Historical and Aesthetic Perspectives

\section{Pierre-Mong Lim}

\section{(2) OpenEdition}

1 Journals

Édition électronique

URL : https://journals.openedition.org/gradhiva/5659

DOI : 10.4000/gradhiva.5659

ISSN : 1760-849X

Éditeur

Musée du quai Branly Jacques Chirac

\section{Édition imprimée}

Date de publication : 24 mars 2021

Pagination : 176-191

ISBN : 978-2-35744-132-3

ISSN : 0764-8928

\section{Référence électronique}

Pierre-Mong Lim, «La part chinoise de Wifredo Lam. Perspectives historiques et esthétiques », Gradhiva [En ligne], 32 | 2021, mis en ligne le 02 avril 2021, consulté le 25 mai 2021. URL : http:// journals.openedition.org/gradhiva/5659; DOI : https://doi.org/10.4000/gradhiva.5659

(c) musée du quai Branly 


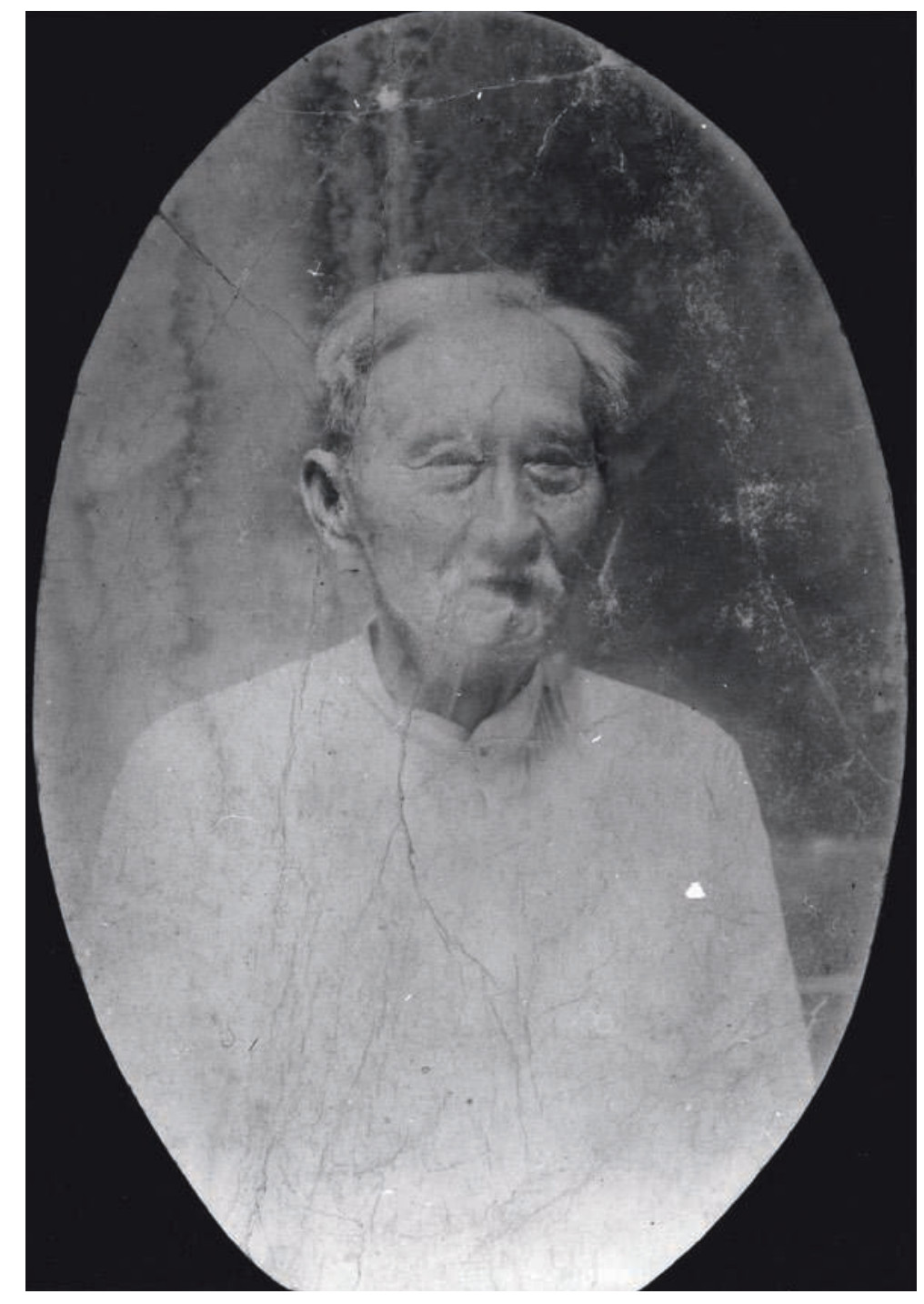

174 


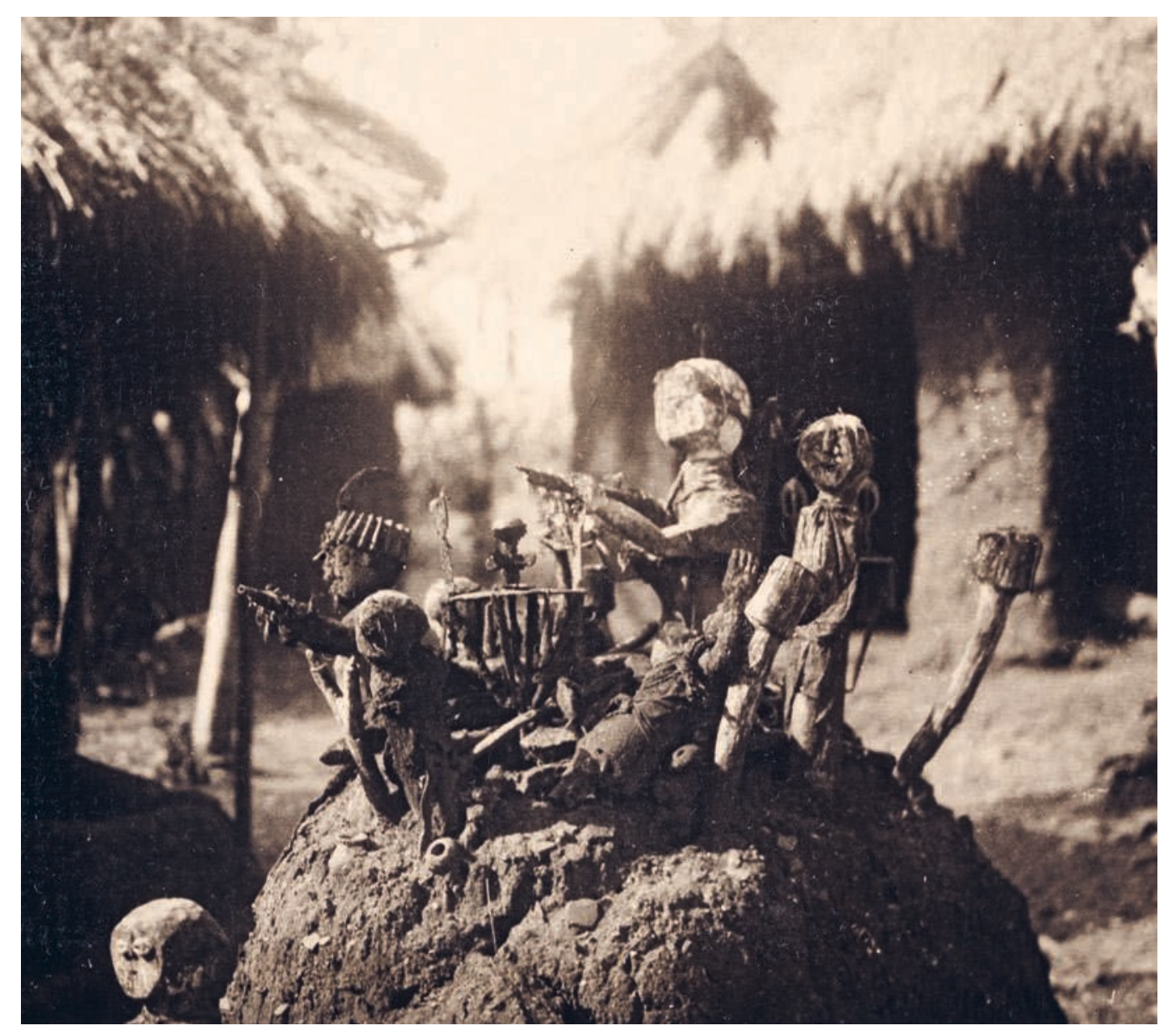


Études et essais

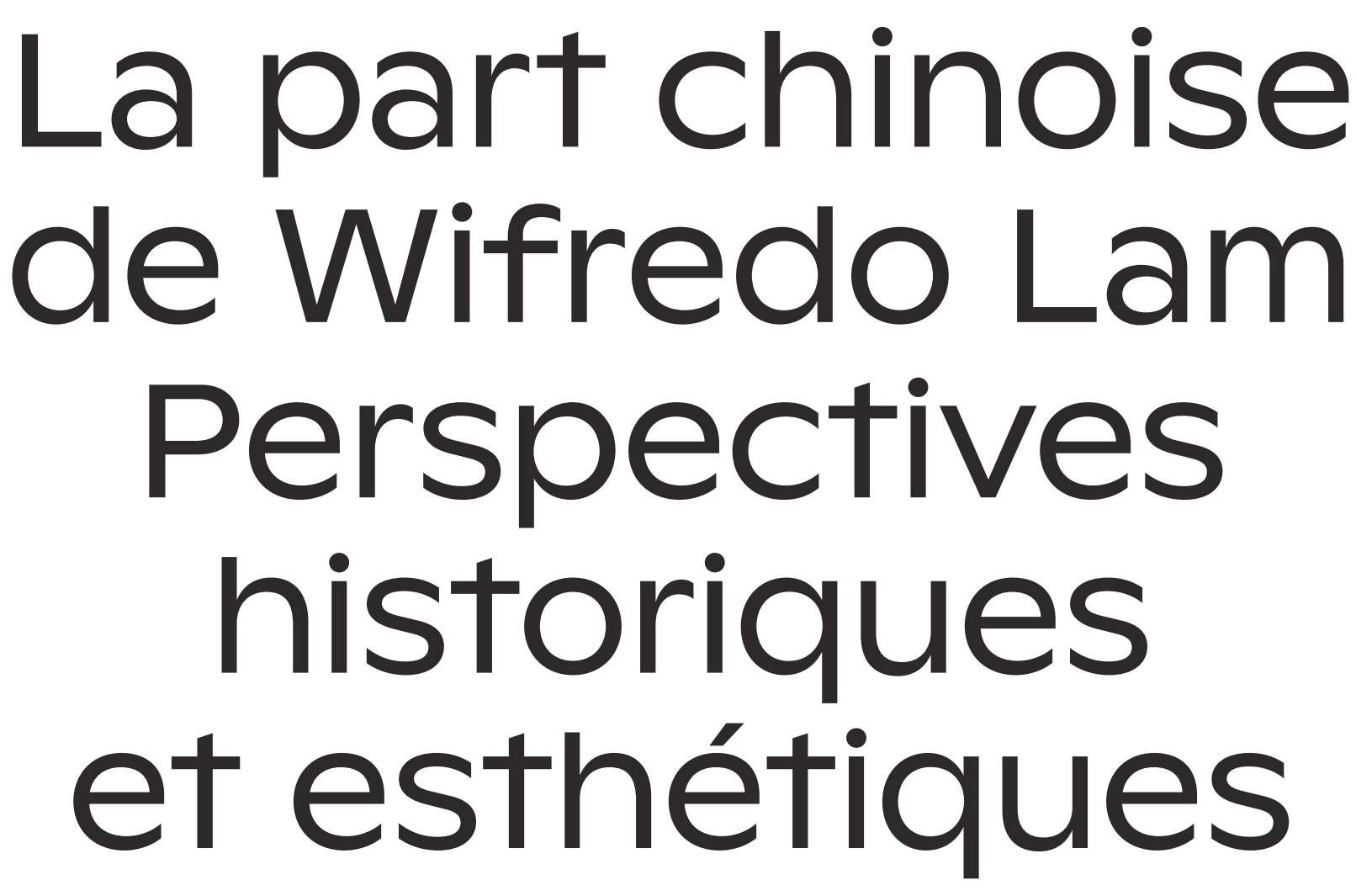

Mots clefs:

La Jungle, peinture lettrée, créolité, modernité, migration des coolies chinois.

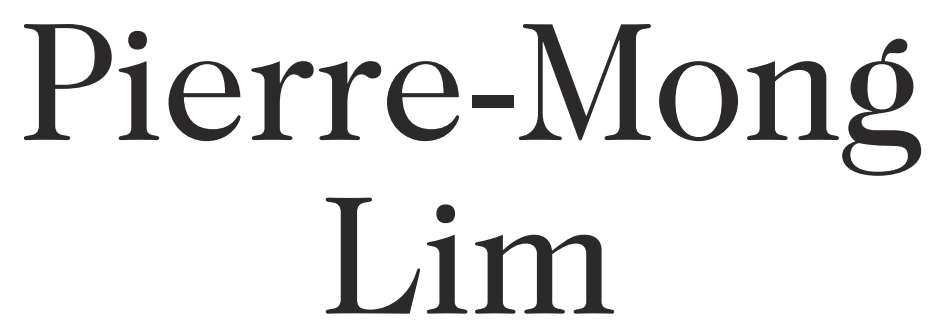


Si le métissage complexe dont est issu Wifredo Lam est connu, le rapport de son œuvre avec ce que l'on peut appeler sa «part chinoise» reste obscur. Nous proposons ici de réfléchir aux liens qui pourraient être établis entre une certaine idée de l'expérience picturale attachée à la peinture lettrée, telle qu'elle s'élabore au début du xx ${ }^{\mathrm{e}}$ siècle, et l'expérience de Lam. Nous verrons qu'il existe un commun ancrage dans les problématiques modernistes touchant à la conception de la représentation et la conduite créatrice. Par cette méthode transversale, nous essayons d'ouvrir un nouvel horizon dans l'interprétation de la peinture de Lam, notamment La Jungle, qui, réexaminée dans cette perspective, éclaire la teneur de cette part chinoise et, de ce fait, de son métissage.

Une vieille photo de 1920, le portrait au crayon que Wifredo Lam dessina à 20 ans sur un bout de papier nous ont fait connaître le visage de Lam Yam, le père du peintre, dont il parlait ainsi:

Si je pense à mon père, le Chinois, je me rappelle un homme très sage, très secret. II réfléchissait en maniant un éventail [...]. Avec lui, il apportait la mémoire de toutes sortes de paysages - sibériens, mongols, tartares - le drame de l'Asie et de la mer de Chine. Dans ses yeux se levait le soleil d'une île convulsive qui luttait pour sa liberté.

(Fouchet 1998 [1976]: 36)

De là naît une curiosité pour cette part chinoise de Wifredo. Elle ne transparaît pas dans son œuvre, même si de rares auteurs ont pu suggérer l'influence de la peinture chinoise sur son travail, sans véritablement préciser en quoi elle consistait, sans définir ni histori-

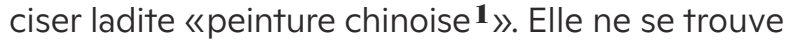
pas non plus dans une identité politique, le lien avec la Chine apparaît même en négatif, puisque Wifredo, qui héritait par son père de la nationalité chinoise (dispositif juridique inédit conçu par le gouvernement de I'Empire des Qing sur le point de s'effondrer) y renonce en 1923 quand, à 21 ans, il acquiert la nationalité cubaine ${ }^{2}$. Lam Yam semble avoir été lié aux activités outre-mer du parti nationaliste de Sun Yat Sen, mais son fils, comme beaucoup parmi la deuxième génération de migrants, reste étranger au phénomène de «nationalisme à longue distance», tel qu'il se manifeste chez les Chinois de Cuba.

D'autant plus que, sur l'île, comme les autres enfants nés de pères chinois et de mères africaines ou mulâtres, il est rangé parmi les mestizos. Si certains d'entre eux espèrent, par cette classification, appartenir pleinement à une jeune nation cubaine transculturelle et transraciale, race et couleur de peau continuent à définir les appartenances et les différents degrés de la «cubanité». (López 2013: 210-213)

Ainsi, Wifredo, se souvenant de sa jeunesse cubaine, évoquera son métissage d'abord comme un malaise: «Je n'étais considéré ni comme un Africain, ni comme un Chinois, ni comme un Espagnol ou un Créole, car je suis métissé avec plusieurs races» (Xuriguera 1974: 9). L'année même où il change de nationalité, en 1923, il part pour l'Espagne, début d'une errance qui ne connaîtra pas de fin, au cours de laquelle la seule identité en formation qui va véritablement compter est celle du peintre.

Car rappelons-nous les mots de Michel Leiris:

Mais ces détails généalogiques, qui montrent en Wifredo Lam le fruit d'un de ces métissages complexes dont les Antilles ont été le théâtre, ont-ils une importance? Assurément non, si l'on cherche dans cette donnée, prise sous l'angle biologique, quelque explication de l'envoûtement très particulier qu'exerce sa peinture [...]. Toutefois, ces
1. Madeleine Rousseau parlait d'«une technique inspirée par la simplicité des peintures chinoises » (Rousseau 1948: 591), et Jacques Leenhardt voit cette influence dans le travail du pinceau (Leenhardt 2009 245). Signalons également la proposition singulière du chercheur japonais Hiroshi Murata sur les liens entre l'alchimie chinoise et la peinture de Lam (Murata 2005). Notons que la dernière grande rétrospective de l'œuvre de Lam, bien que fort riche en maints aspects, n'a pas apporté d'éclaircissement nouveau sur ce point (David 2015)

2. Sur la question de la nationalité de Lam Yam et de son fils, voir la biographie en espaǵnol consacrée au peintre par Núñez Jiménez (1982), dont certains éléments sont repris dans Hu-Dehardt 2013: 98-99.

Notons ici que l'identité politique chinoise connaît un profond bouleversement à la fin du XIX ${ }^{e}$ siècle: alors que traditionnellement il était inconcevable que des " communautés chinoises " puissent exister en dehors des frontières de l'Empire, l'autorité impériale des Qing est forcée de réimaǵiner le lien politique avec ses sujets extérieurs, suite aux traités internationaux (dits «inégaux»), internationaux (dits «inégaux »),
comme celui de Nankin en 1842 , comme celui de Nankin en
et à l'émigration en masse de travailleurs qui fourniront les funestes contingents 
de coolies, main-d'œuvre qui part remplacer les esclaves africains libérés après les différentes abolitions dans les colonies européenn notamment dans les Caraïbes. notamment dans les Caraïb
Le destin de Lam Yam est intimement lié à cette histoire, nous y revenons plus loin. C'est en 1909 qu'est adoptée la loi sur la nationalité, basée Cla le principe dujussangee sur le princelpe dujus sanguinis. Voir notamment: Wang 199 [1981] ; Zhuang 2013;

Pann 2000.

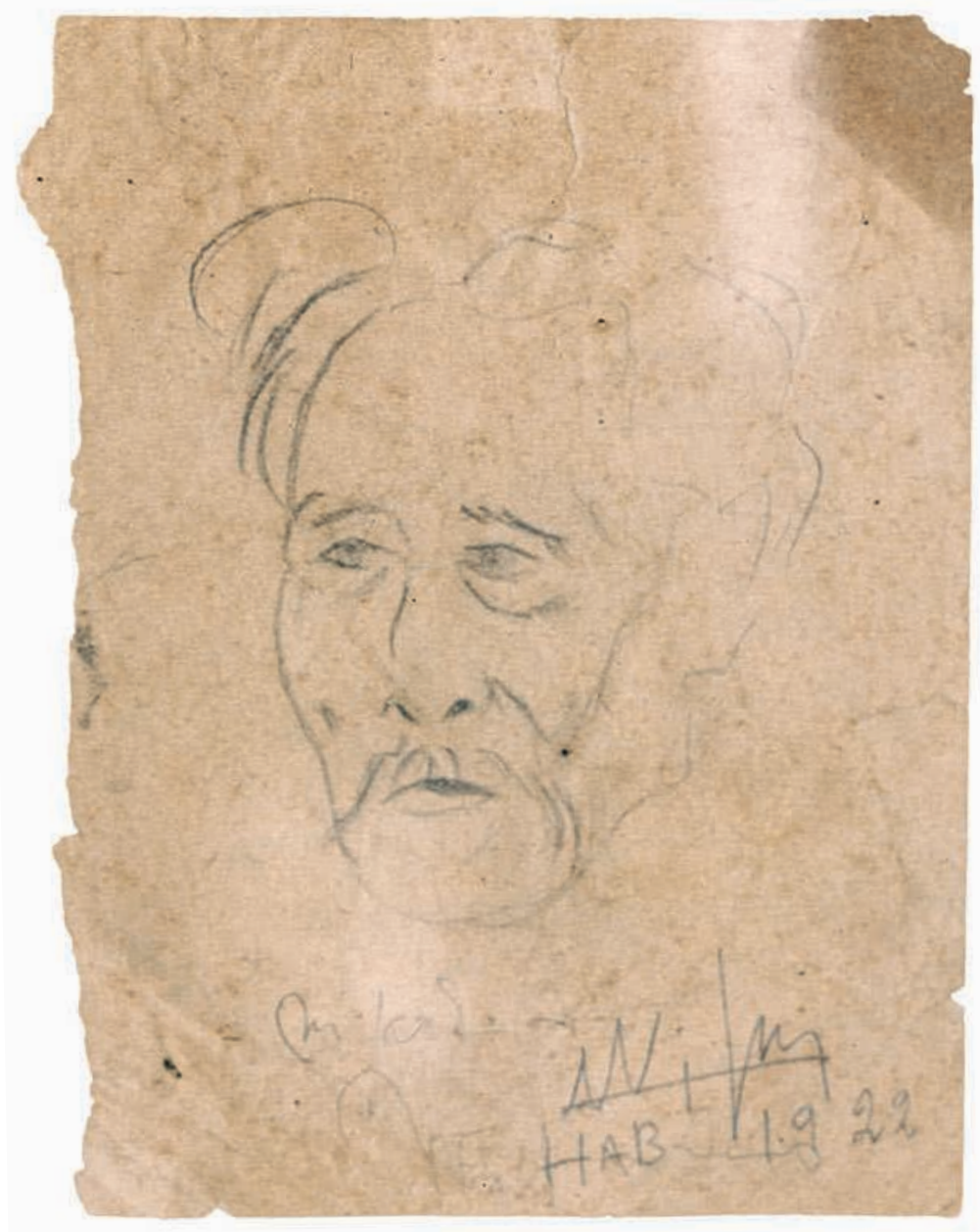

Portrait de Lam Yam, 1922. Crayon sur papier, 14,5 ×11 cm. Collection privée, Paris @ Paris, ADAGP, 2021.

données - apparemmentanecdotiques - sur la généalogie de l'artiste sont plus intéressantes [...] pour peu qu'on les envisage, non sous l'angle du «sang», mais en tant qu'indices de conditions très spéciales dans lesquelles sa jeunesse s'est déroulée.

(Leiris 2011: 425)

Il s'agirait donc d'approfondir l'œuvre du peintre à la lumière de la complexité de ces métissages et des questionnements qu'ils suscitent, d'en proposer, peut-être, une interprétation nouvelle qui, en ouvrant d'autres horizons, ne supplante pas les précédentes mais s'y mêle. Ainsi, ce que nous nommons ici «part chinoise», faute de mieux, reste inséparable des autres parts qui constituent Wifredo Lam, notamment le lien très fort qui l'attache aux Afro-Cubains (entre autres, par sa mère et sa marraine, Mantonica Wilson). Nous écarterons d'autant mieux les réductions biologiques, ethniques ou nationales que nous envisagerons, tout d'abord, la question d'un point de vue esthétique, en laissant de côté les codes esthétiques chinois, visiblement absents de la peinture de Lam, pour prêter attention à la seule conduite créatrice, au rapport du sujet à son œuvre. Dans ce cadre, nous reviendrons sur les propositions d'un peintre nommé Chen Shizeng, qui, dans les débats picturaux qui agitent la République de Chine des années 1920, rechercha un commun rapport entre peinture lettrée et courants modernistes européens; nous verrons se dessiner des correspondances et une préoccupation similaire pour le travail intérieur du peintre. Certes, selon toute vraisemblance, Lam n'a pas eu connaissance de cette théorie lettrée moderne, pourtant il épouse les mêmes courants modernistes européens, presque simultanément en Espagne. À partir de là, nous proposons d'éclairer les deux démarches I'une par l'autre: comment Lam, par son expérience, dépasse la posture lettrée et indique le sens véritablement moderne de l'intériorité recherchée. Par ailleurs, nous verrons dans quelle mesure cette compréhension actualisée de l'intériorité lettrée permet d'aborder à nouveaux frais le langage pictural de Wifredo. C'est dans son célèbre tableau, La Jungle, que nous examinerons comment tout est ressaisi, la dimension esthétique ouvrant à une dimension historique proprement cubaine, où le métissage de Lam s'enrichit d'une signification, peintre lettré immédiatement créole.

\section{PEINTURE LETTRÉE, MODERNISME ET RÉSONANCES EN EUROPE}

La transformation de l'imaginaire politique, au moment de la chute de l'Empire Qing et de la révolution républicaine de 1911, ne se traduit pas uniquement par l'élaboration d'une identité nationale et une recon- 
La part chinoise de Wifredo Lam, perspectives historiques et esthétiques

Pierre-Mong Lim

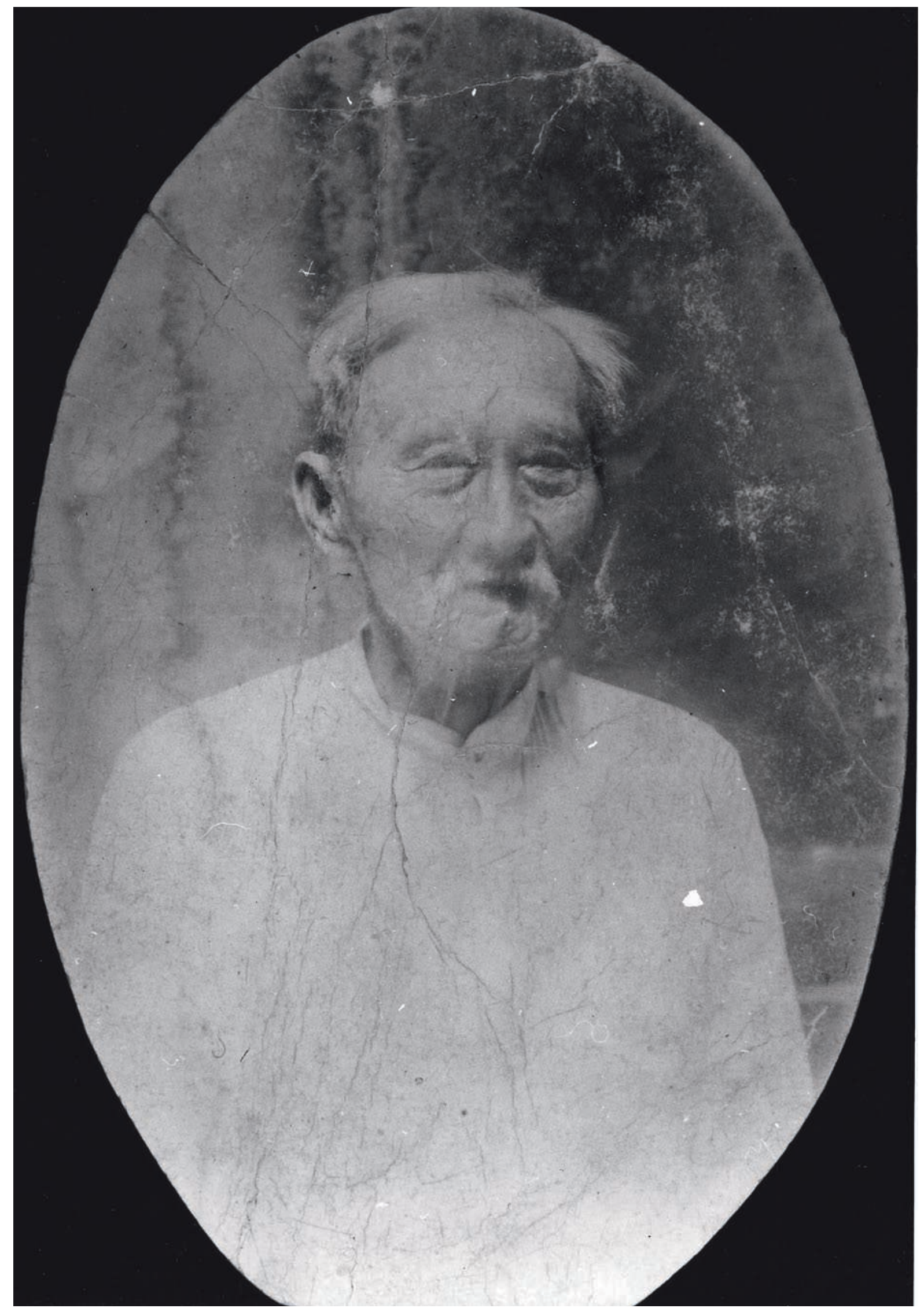

Photographie de Lam Yam, s.d. Collection privée, Paris. 
3. À l'issue du traité de Versailles, le transfert des possessions allemandes de certaines provinces chinoises au bénéfice du Japon provoqua un vaste mouvemen de protestation de la part des étudiants. Ce «mouvement du 4-Mai», comme on l'appela, dépassa vite les revendications politiques pour se transformer politicues par se transhorme ionner la société, notamment la langue la littérature, la peinture.

4. Sauf indication contraire, toutes les traductions sont de l'auteur. naissance juridique que l'on accorde par exemple à des émigrés tels que Lam Yam; elle s'inscrit aussi dans un processus de transformation du rapport au réel, à l'intérieur duquel la place de l'artiste, du sujet créateur, est fortement remise en question. L'intensité de ces questionnements atteint son paroxysme lors du mouvement du 4 mai 19193 , quand, sous les deux mots d'ordre «nationalisme» et «raison», les tenants d'un certain progressisme tentent de penser la modernité chinoise sous toutes ses formes, notamment dans les arts et les lettres (Chow 1967; Schwartz 1972; Schwarcz 1986).

À l'intérieur de ces très nombreux débats, attirons l'attention sur la controverse connue autour du statut de la «peinture lettrée», suscitée par un écrit du vieux réformateur Kang Youwei. En 1918, dans un court texte qui tient lieu de préface à la «Collection de peintures du Pavillon luxuriant», Kang constate la déchéance contemporaine de la peinture chinoise. Il commence par rappeler les anciennes définitions de la «peinture» afin de prouver que celle-ci était originairement comprise comme la restitution des formes: «Le Erya définit: "la peinture comme la forme", le Guangya: "la peinture comme la ressemblance" [...] le Classique des documents: "c'est employer les cinq couleurs pour créer une image $\mathbf{4}^{\prime \prime} \gg$ (Kang 1998 [1918]: 3). En retraçant schématiquement l'histoire de cette peinture, il attribue son fourvoiement aux maîtres de la peinture lettrée incarnée par l'école des Quatre Wang, à l'origine, dit-il, de la tendance qui a éloigné le peintre de la réalité, et plaide alors pour un retour au réalisme par lequel la peinture chinoise rejoindrait la peinture occidentale.

II est suivi, un an plus tard, en 1919, par l'une des figures les plus importantes de la révolution littéraire du 4-Mai, Chen Duxiu, qui, bien que divergeant politiquement de Kang, appelle dans les mêmes termes à une «révolution de l'art». II s'en prend avec violence aux modèles sclérosés d'une peinture lettrée qui, en brimant toute autre forme d'expression, s'est rendue responsable de la décadence des arts en Chine, il exhorte donc les peintres à abandonner, suivant l'exemple des poètes modernes, la copie des anciens maîtres et à renouveler ainsi leur vision en revenant à un rapport réaliste avec les choses du monde. Voici ce qu'il écrit:

Il nous faut tout d'abord mettre à bas la peinture des Quatre Wang si I'on veut réformer la peinture chinoise, parce qu'une telle réforme rend indispensable l'adoption de l'esprit réaliste de la peinture occidentale. Quelle en est la raison? [...] [L]es peintres doivent aussi avoir recours au réalisme, ils pourront alors de même donner libre cours à leur génie, peindre leurs propres peintures et non pas se traîner derrière les stéréotypes des anciens.

On a pour habitude de tenir la peinture de Wang Shigu [Wang Hui] pour l'incarnation de la grande synthèse de la peinture chinoise: je dis pour ma part que cette peinture-là n'est que le résultat de la mauvaise peinture de la clique des Nizan, Huang [Gongwang] et Wen [Zhenming]. [...] Surle plan technique, non seulement la peinture de l'école des Wang est loin d'égaler ses modèles des Song et Yuan, mais elle est dépassée par ses contemporains tels que Wu Mojin (Wu est le disciple de Tianzhu, sa technique a reçu l'influence de la peinture occidentale) [...] si on ne [la] renverse pas pour importer le réalisme, ce sera le plus grand obstacle pour réformer la peinture.

(Chen 1998: 4)

Cette ligne était représentative des tenants d'une «modernisation» de la Chine, les intellectuels tels que Lu Xun, Cai Yuanpei, ou les praticiens comme Xu Beihong revendiquaient tous avec plus ou moins de force l'introduction du réalisme occidental pour régénérer la peinture chinoise (Andrews et Shen 2006: 4-9).

Mais en Janvier 1921, le peintre et théoricien Chen Shizeng publie dans la revue de la Société pour la recherche des méthodes de peinture (Huafa yanjiuhui 畫法研究會), qui vient de voir le jour à l'université de Pékin, un article intitulé «La valeur de la peinture des lettrés». On peut certes le lire comme une réponse aux précédentes attaques de Chen Duxiu et de Kang Youwei contre la «clique des Quatre Wang», mais en prenant la défense de cette peinture, Chen Shizeng propose en fait une profonde réflexion sur les problématiques que son époque pose à l'art, sur le rapport sujet-objet, et tente d'indiquer une alternative à ce que ses contemporains comprennent comme étant la modernité (Chen 2010: 141-147).

II prend tout d'abord le contre-pied des tenants du réalisme: certes, dit-il, selon leurs critères la peinture lettrée paraît un barbouillage maladroit, dont les formes sont inexactes. Mais le lettré n'est pas un photographe qui doit parfaitement restituer un objet, sa peinture est l'expression, le prolongement de l'individualité et de sa perception. Chen en appelle aux six règles classiques établies par Xie He, mais surtout au jugement sarcastique du plus célèbre des peintres lettrés, Su Shi: «Celui qui valorise la ressemblance formelle en peinture, je le considère attardé dans l'enfance.» (Ibid.: 142) Par-là, il ne se contente pas de mortifier ses adversaires réalistes, il explique que si cette peinture accorde plus d'importance à l'esprit, c'est que les hommes de qualité savent que la forme est imparfaite, alors que l'esprit, lui, est parfait. Tel est le modèle intérieur que propose le lettré. Cependant, la contreattaque ne serait rien d'autre qu'une banale défense conservatrice si Chen n'affirmait pas en même temps que, dans la mesure où la peinture lettrée ne recherche pas la reproduction fidèle de l'objet mais le vécu sensible de l'artiste, elle prouve qu'elle est «en avance» sur son temps: «La peinture des lettrés ne recherche pas la ressemblance avec la forme, c'est une peinture tout à fait avancée», écrit-il (ibid.: 146). Voilà qui est surprenant: ce que certains considèrent comme 
La part chinoise de Wifredo Lam, perspectives historiques et esthétiques Pierre-Mong Lim

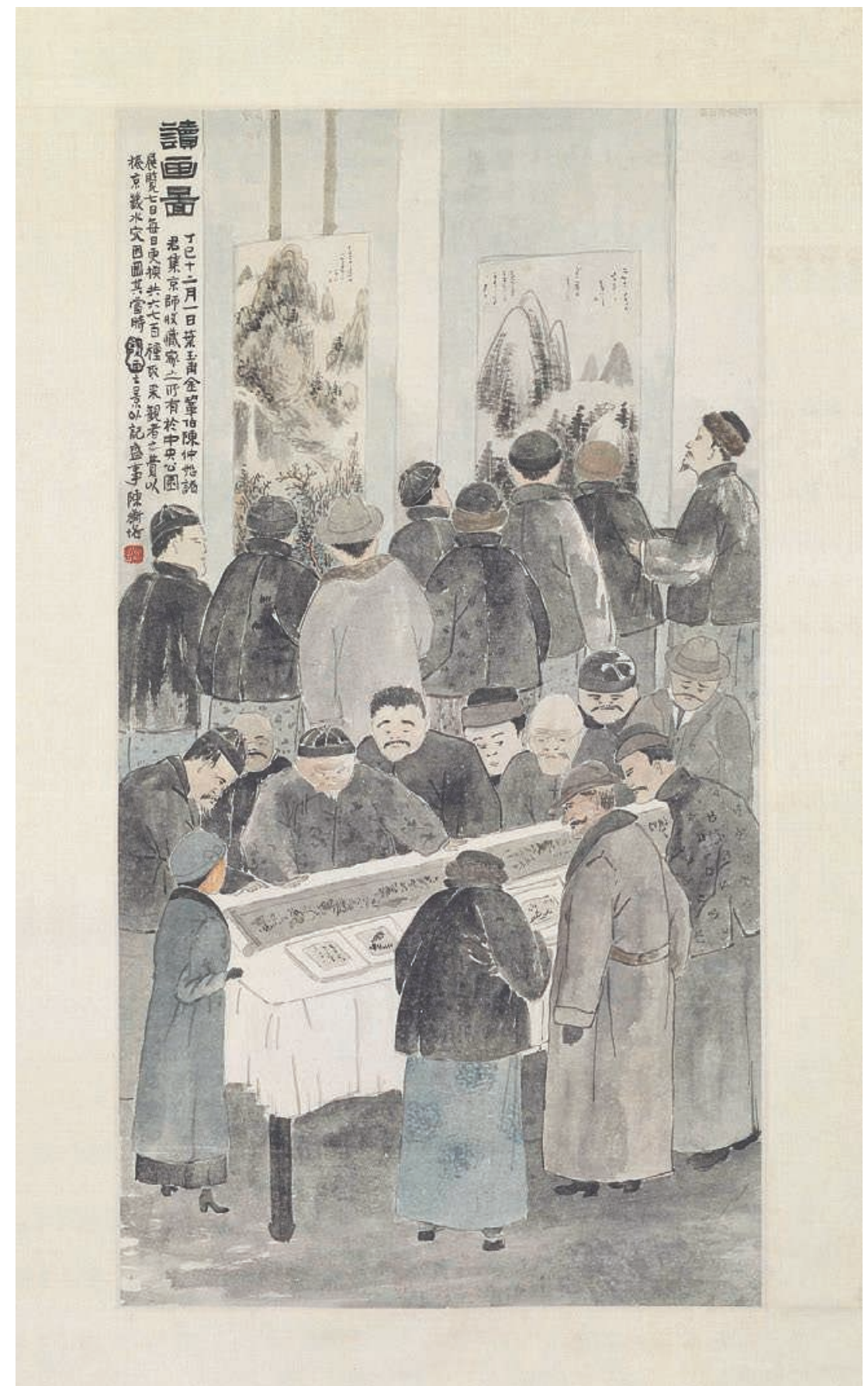

Chen Shizeng 陳師曾,Viewing Paintings at an Exhibition 讀畫圖 [Duhua tu], 1917

Parchemin suspendu, encre et couleur sur papier, $87,7 \times 46,6 \mathrm{~cm}$. The Palace Museum, Pékin, Chine. Droits réservés. 


\section{Études et essais}

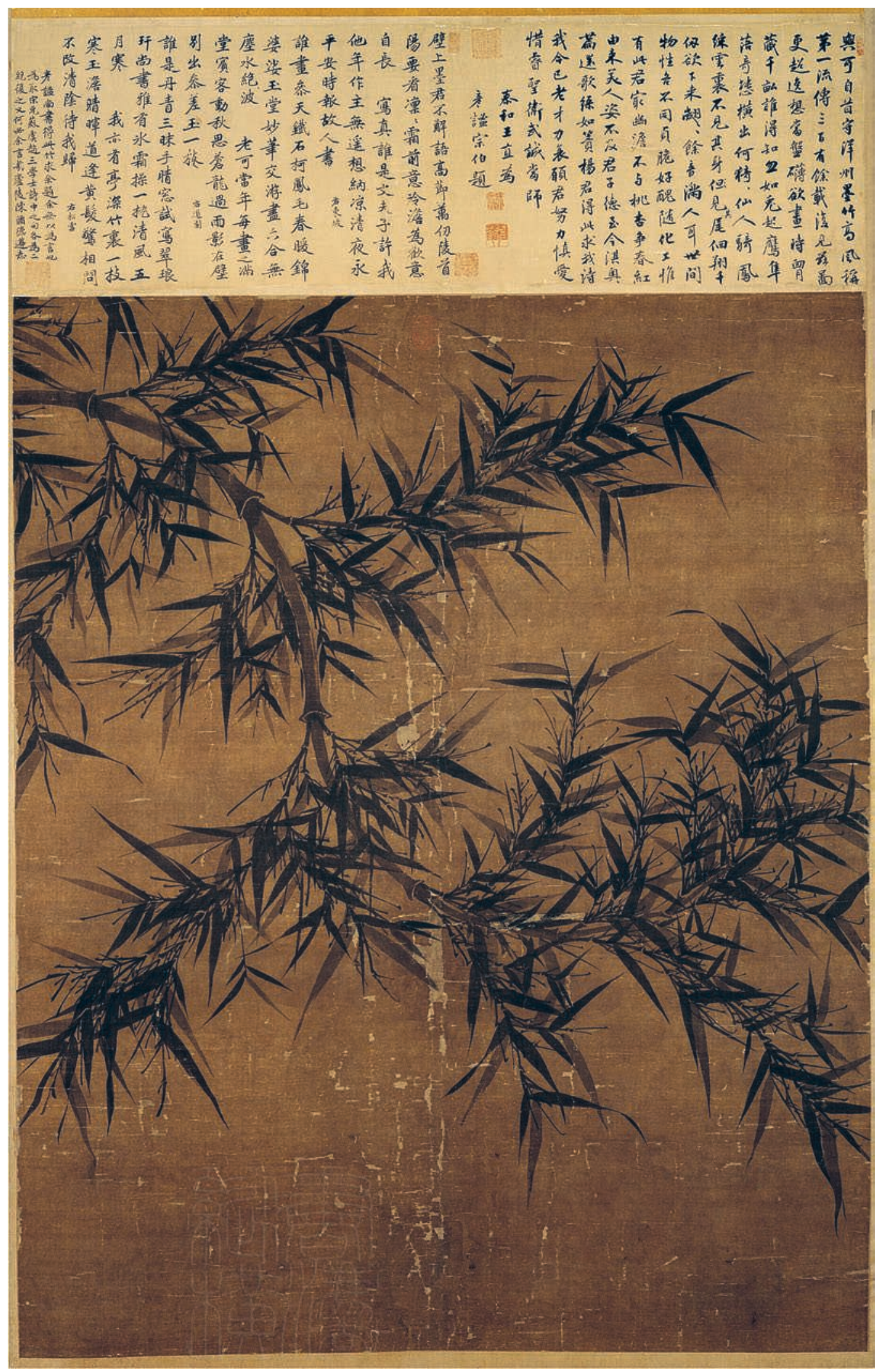

Wen Tong 文同 (Yuke), Bambous à l'encre 墨竹圖, encre sur soie, 131,6 × 105,4 cm. (c) Musée National du Palais, Taipei. Droits réservés. 
I'arriération même, et que d'autres chérissent au contraire par nostalgie de l'ordre ancien, Chen en fait la pointe de la modernité. Ces règles anciennes auraient déjà posé les fondements d'une expérience, d'une subjectivité créatrice «avancée» dans l'histoire de l'art, bien plus avancée que la modernité réduite du réalisme qui fascine tant ses contemporains. Mais sur quoi Chen s'appuie-t-il pour oser une telle affirmation? C'est le point de sa réflexion qui nous semble le plus important: la peinture lettrée, en permettant à l'homme de descendre dans les profondeurs de la subjectivité, rejoint les tendances les plus récentes de la peinture occidentale. Vers la fin de l'article, on lit ceci:

\begin{abstract}
Oui, nous pouvons dire que la peinture occidentale s'approche au plus près de la ressemblance formelle! Depuis le $x I X^{e}$ siècle, suivant les principes de la science, cette peinture a poursuivi ses recherches sur la lumière et la couleur, elle a fait de l'objet son domaine, tandis qu'elle abandonnait l'expérience vécue. Mais récemment, le postimpressionnisme est allé à rebours de cette tendance, il a cessé de privilégier l'objet pour se concentrer sur le sujet. Rejoints par le cubisme, le futurisme et l'expressionnisme, ces bouleversements dans la pensée suffisent à montrer que la ressemblance formelle n'épuise pas tout l'art, et qu'il doit y avoir d'autres recherches à mener.
\end{abstract}

(Ibid.: 147, nous soulignons.)

Chen s'était intéressé très tôt aux mouvements contemporains de la peinture occidentale, comme en témoigne sa traduction, dès 1912, d'un article du peintre japonais Kume Keiichirô, intitulée: «Situation actuelle dans le monde de la peinture européenne» (Ouxi huajie zuijin zhi xiankuang 歐西畫界最近之現狀). Mais dans l'article de 1921 (notamment la version révisée $\mathbf{5}$ ), il franchit un pas en suggérant maintenant une communauté d'expérience entre la peinture lettrée et les écoles modernistes: elles rejettent de concert le rapport au monde ramené à des données purement objectives, pour accorder au vécu subjectif une place prépondérante. On peut regretter que Chen ne donne pas plus de détails sur les aspects particuliers de cette correspondance qu'il pense déceler. Mais, dans sa démarche, il faut remarquer que l'esthétique de la «ressemblance formelle» s'inscrit dans une volonté de prise en charge scientifique du monde, caractéristique de l'Occident - ce à quoi aspirent ses contradicteurs -, à laquelle il oppose donc ces mouvements modernistes: ceux-ci, notamment le cubisme, d'une part se sont dégagés de la conception d'une spatialité homogène (la «perspective géométrique», I'ordonnancement du monde et de son espace selon les lois mathématiques, perçu par un œil unique et plat) en portant une attention renouvelée à I'homme dans son rapport au monde sensible, à son «espace psychophysiologique» pour reprendre l'expression d'Erwin
Panofsky (1975: 43). D’autre part, bien que nés en Europe, ils réhabilitent les arts de civilisations différentes en procédant à des emprunts qui, bien que très localisés, semblent apporter des réponses aux problèmes que se pose le modernisme en peinture.

Nous pouvons pousser plus loin la réflexion sur certaines correspondances - esquissant ainsi un premier lien avec la peinture de Wifredo Lam -, car on peut être tenté de rapprocher les propos de Chen de la constatation de Jean Laude sur la réception en Europe des arts des pays colonisés au début des années 1900: «Tantôt cet intérêt pour le sauvage et le primitif est le signe d'un refus de la vie moderne; tantôt il est affecté d'un indice positif, comme appartenance au mouvement moderniste.» (Laude 2006 [1968]: 14) Comme si, de part et d'autre, l'expérience attachée à ces arts non occidentaux consistait en un rejet de la modernité et, en même temps, était en avance sur elle, préfigurait les saillies les plus modernes. Pour être plus précis, on peut se référer au même Laude, qui parlait ailleurs d'une «pensée des rapports» dans les arts plastiques; mettant en relation l'influence des arts africains sur le cubisme et des «idéogrammes chinois» chez un sculpteur tel qu'Henri GaudierBrzeska, il écrivait:

Le problème ne se situe pas au niveau de l'emprunt, ni à celui de l'adaptation [...]. Il se pose comme changement dans les conceptions qui règlent le rapport du peintre à ce qui l'entoure, au niveau même des systèmes de valeurs.

(Laude 1971: 27)

Ce serait, ici, à un même bouleversement des valeurs que participent la peinture lettrée refusant la ressemblance formelle à l'occidentale et les arts de l'Afrique redécouverts par des cubistes soucieux de se détacher de l'imitation du monde visible et d'affirmer la nature autonome des arts plastiques (Kahnweiler 1948: 367-377). Non seulement il existe entre eux le lien négatif d'appartenir à un non-Occident qui refuse des lois de représentation caractéristique de la culture occidentale, mais primitivisme et peinture lettrée, loin de valider un darwinisme transposé à l'histoire de l'art, rendent également possible un retour aux données immédiates des sens, comme une vision épurée, et révèlent à l'artiste de la modernité une expérience ensevelie. En 1915, Carl Einstein (que Lam va d'ailleurs rencontrer plus tard en Espagne) écrit dans son Negerplastik que «l'être humain se transforme toujours et cependant, il tente constamment de préserver une certaine continuité, son identité. Les Européens ont voué à celle-ci un culte excessif. Le Noir qui se sent moins embarrassé par cette subjectivité du moi et qui révère les puissances objectives doit [...] se transformer en elles» (Leenhardt 2009: 55). L'enjeu de l'expérience cachée devient plus précis, c'est l'abolition de la séparation nette entre le domaine de l'objet et celui du sujet par une puissance de transformation, voire de fusion à l'œuvre dans le rapport créateur. Ainsi, comme l'homme africain se perd et se relève dans l'objet qu'il crée (selon l'interprétation
5. Comme le rappellent Andrews et Shen, Chen écrivait dans une première version: «Il est dit que les peintures occidentales se préoccupent de la ressemblance formelle, mais que dire alors des écoles actuelles de la nouvelle peinture qui rompent totalement avec les conventions du passé? Comment voir la ressemblance formelle dans les peintures des écoles futuriste et cubiste?» (Andrews et Shen 2006: 8) 
6. Pour une analyse du contexte général de cette controverse entre les troi auteurs, voir: Panǵ 2007. $42-45$ des milieux modernistes), le peintre lettré de Chen doit s'abolir dans l'objet qu'il peint pour rejoindre un mouvement général, total, qui le dépasse et l'englobe - c'est la vaste pensée de Su Shi sur laquelle il s'appuie; nous y reviendrons.

Cependant, Chen, ne pouvant - ou ne voulant dépasser les références à cette culture lettrée, ne poursuit pas dans la voie qu'il vient d'ouvrir. Notre auteur arrive à la fin de son article sans explorer plus avant ce qu'aurait pu signifier cette subjectivité partagée entre le peintre lettré et le moderniste, ni ce qu'impliquait cette commune subjectivité découverte dans l'élan créateur guidé par la perception intérieure; on relève certes une référence à l'esthétique de l'empathie, Einfühlung, que serait censée accomplir la peinture lettrée, ainsi que l'emploi, pour évoquer l'expérience picturale, d'un terme comme tixian 體驗, qui a littéralement le sens d'une expérience vécue où le corps est engagé, mais l'auteur ne s'attarde pas (Chen 2010: 147). Chen se borne à énumérer quatre éléments qui composent cette peinture lettrée: moralité, érudition, inspiration, réflexion (renpin 人品, xuewen 學問, caiqing 才情, sixiang 思想 [ibid.], qui toutes ressortissent du domaine de l'intériorité, mais sont ramenées à l'élévation morale très convenue du lettré. Chen se réfugie finalement dans les hauteurs d'une pratique culturelle validée par la supériorité d'une classe (parce que le contexte du débat le veut; il y a alors une polarisation des visions de la culture chinoise 6 ), laissant ainsi inachevée «l'avancée» de la peinture lettrée.

À quel autre type d'intériorité aurait pu se référer Chen, lui qui pensait découvrir dans les mouvements contemporains modernistes la même expérience picturale qu'il défendait? II ne semble pas avoir eu connaissance du premier groupe des surréalistes qui trouvèrent une illustration plastique de leurs aspirations dans le cubisme et les arts dits primitifs (en 1921, l'année même où paraissait cette défense de la peinture lettrée, André Breton publiait ses Champs magnétiques). E† pourtant, Breton, quelques années plus tard en 1928, tient dans Le Surréalisme et la peinture des propos qui touchent de près aux préoccupations de Chen: il rejette avec force, lui aussi, la peinture réduite à n'être que la pâle copie de l'apparence de l'objet, il s'agit pour lui de libérer l'œuvre de sa soumission à la représentation, à cette «conception très étroite de l'imitation, donnée pour but de l'art» (Breton 1965 [1928]: 14). E† l'on remarquera que cette réflexion prend place dans un contexte de modernité technique déjà globale, où la photographie qui permet la reproduction parfaite de l'objet questionne «la raison d'être» de la peinture, chez Breton comme chez Chen (ibid.: 52; Chen 2010: 141). Or, Breton appelle les peintres à rechercher dans un «modèle intérieur» la puissance qui les fera accéder à une profondeur encore jamais atteinte dans le rapport au réel: «L'œuvre plastique, pour répondre à la nécessité de révision absolue des valeurs réelles sur laquelle aujourd'hui tous les esprits s'accordent, se référera donc à un modèle purement intérieur, ou ne sera pas» (Breton 1965 [1928]: 15). À partir d'une même préoccupation esthétique que celle de notre peintre lettré, et cela jusque dans les termes employés, Breton et son groupe de surréalistes vont cependant découvrir une intériorité insoupçonnée par Chen dans l'expérience qu'ils feront de l'automatisme - si importante pour Lam par la suite. Et il est permis de penser que cette aspiration à l'intériorité du vécu fut plus pure du côté de l'automatisme, parce que moins directement soumise aux préoccupations de classe, de nation voire de race auxquelles succomberont les propositions de Chen, et plus largement la narration moderne de la peinture lettrée qui restreindra ainsi l'expérience intérieure là où elle devait, au contraire, s'ouvrir à tout sujet créateur qui opère la descente en soi-même, là où tout communique, où tout s'effondre et se recompose.

\section{LE JEUNE LAM}

Cette mise en contexte générale doit à présent nous amener à réexaminer la peinture du jeune Lam, dans laquelle tout ce que nous avons vu jusqu'ici va d'abord être ressaisi. Nous sommes donc en 1923 quand Wifredo Lam, désormais Cubain, arrive à Madrid. Rappelons qu'au commencement, c'est pour y être formé dans l'atelier d'un peintre académique, mais Lam se détourne rapidement d'une peinture classique à laquelle il se sent étranger. Son séjour espagnol, qui dure jusqu'en 1937, a ceci de remarquable pour notre propos que les doutes éprouvés par le jeune peintre quant à la manière classique le portent, d'abord vers d'autres maîtres anciens (Bosch, Goya), puis très vite vers les courants modernistes qui se développaient depuis quelques décennies. En témoignent certains tableaux de jeunesse, où cette influence est visible par certains thèmes ainsi que dans la façon dont Lam organise l'espace. «Au début des années 1930 différents courants du modernisme européen commencèrent à transparaître dans les travaux de Lam: il adapta les tendances figuratives post-cubistes similaires à celles de l'école de Paris des années 1920 et aux tendances contemporaines en Espagne», analyse Lowery Stokes Sims, plus précisément, il signale par exemple un tableau daté de 1932, Paisaje III, dont le traitement fragmenté rappelle Cézanne et certains aspects du fauvisme (Sims 2002: 13). De plus, il faut souligner que l'intérêt qu'il nourrit alors pour ces recherches picturales vient d'une attention aiguë portée au vécu intérieur, le sien propre, en prise avec l'histoire. Comme l'écrit Leenhardt, il semble «avoir trouvé dans la synthèse du cubisme analytique, une manière de figurer qui convient à son expérience personnelle d'un monde fragmenté et contradictoire» (Leenhardt 2009: 53), où la décomposition du point de vue est avant tout décomposition de I'homme qu'empêchent de se rassembler sur lui-même ses drames personnels (la perte de sa première femme et de son enfant) et les conditions politiques particulières à l'Espagne déchirée de cette époque.

En outre, si ce refus opposé aux règles classiques de composition le situe dans la lignée des mouvements européens de la fin du xIx siècle, il le prépare en 
quelque sorte à accueillir le travail de Picasso, dont les œuvres, qu'il voit une première fois exposées à Madrid en 1936 avant de rencontrer le maître à Paris, lui font une impression très forte. De Picasso, Pierre Mabille, qui situait Lam au bout de cette course à la destruction des règles classiques, dit qu'il «oscillera désormais entre cette tradition [de la perspective] et la volonté de la détruire [...]. Il est à la fois le continuateur de Cézanne, l'architecte et l'allié des sauvages dont les forces intactes menacent de triompher du vieux maître fatigué»» (Mabille 1989 [1938-1951]: 128). Le départ d'Espagne va ouvrir un nouvel horizon: de 1937 à 1940, durant la période dite «parisienne», les œuvres produites par Lam sont profondément marquées par l'enchevêtrement du formalisme cubiste et de la plastique des objets africains. On ignore à quelle date exactement il entre en contact avec la statuaire africaine (sa rencontre avec Picasso en 1938, dans l'atelier parisien de la rue des Grands-Augustins, est connue; celui-ci, qui était passé par bien d'autres «périodes» depuis, demanda à Michel Leiris d'initier le jeune peintre cubain aux cultures d'Afrique ${ }^{7}$ ). Quoi qu'il en soit, ces statues et ces masques lui inspirent alors des moyens plastiques nouveaux qui se différencient de la figuration occidentale traditionnelle. C'est à ce moment-là que l'on voit apparaître un certain nombre d'éléments empruntés à ces arts africains, qui vont durablement former une partie de son vocabulaire pictural. Ces éléments sont reconnaissables, d'une part, dans les «citations» directes que fait le peintre, c'est-à-dire dans les analogies formelles qui sont substituées aux modèles conventionnels - très visibles par exemple dans le traitement des visages dont les traits sont ramenés à ceux des masques. Mais ils sont également présents, d'une façon peutêtre plus profonde, dans l'assimilation des recherches cubistes qui, comme on l'a vu, ayant pris l'art africain comme une référence plastique à part entière, l'ont déjà totalement intégré au développement de leurs propres formes. En réalité, il est difficile - et vain - de distinguer entre les influences dans les figures que Lam peint à cette époque, néanmoins on les reconnaît sans mal: elles sont pour la plupart absolument frontales, leurs visages, membres et bustes sont structurés en bandes chromatiques, sans modelé des ombres; les formes sont réduites à l'expression géométrique la plus épurée, par exemple la charpente rectangulaire des corps, les simples ronds de couleur pour les seins, I'ovale pur et vide des visages. Sans doute faut-il donner raison à Michel Leiris lorsqu'il estime que l'essentiel de la peinture de Lam est déjà là, dans cette correspondance entre une fusion historique de différents courants esthétiques et la convergence d'exigences intérieures au peintre: sa volonté de «s'élever au-dessus du circonstanciel» et sa fascination pour la cosa negra (Leiris 2011: 431).

Mais un autre élément décisif viendra s'ajouter et participer à la transformation de la peinture de Lam dans les années 1940: la pratique de l'automatisme avec Breton et les surréalistes. Si Lam n'a jamais fait officiellement partie du groupe, cela ne l'a pas empêché de faire siennes les pratiques surréalistes. II les met en œuvre dans les peintures réalisées à son retour à Cuba, et notamment la célèbre Jungle, dont les personnages, raconte-t-il, «sont issus de l'automatisme de la pensée [...]. La Jungle, elle, a été engendrée par un retour aux sources originelles de la nature tropicale, elle est le fruit d'une intuition intellectuelle. [...] Mes tableaux sont le reflet [de mon existence], ils sont une confession intime de mon existence, ils ont une relation très profonde avec "I'intérieur" et le déroulement de mon enfance. André Breton dans son premier Manifeste voulait pénétrer l'essence de la création et soulignait que le comportement psychologique de l'homme, acquis après sa naissance, apparaissait nettement dans son épanouissement créatif. L'automatisme découlant de ces données» (Xuriguera 1974: 9).

Parvenus à ce stade, nous voyons donc que la peinture de Lam s'est constituée selon les problématiques d'un modernisme européen qui, guidé par le refus des principes de la représentation classique, s'est attelé à la recherche d'un «modèle intérieur» qui puisse donner naissance à une forme nouvelle (que ce soit par l'art africain ou l'automatisme). Sans le savoir, il partage ainsi les mêmes préoccupations qu'un Chen Shizeng, mais, lui, ne reculera pas pour s'abriter derrière une quelconque tradition. II va, par un cheminement qui lui est propre, offrir un développement inédit à ces acquis modernistes, et rejoindre, en même temps, selon notre interprétation, l'expérience profonde du peintre lettré.

\section{« LA JUNGLE 》: UNE INTERPRÉTATION}

La Jungle peut donc être présentée comme une vaste synthèse: celle des acquis du modernisme (Tronche 2010: 25), celle des choix thématiques et iconographiques propres à Lam depuis 1942 (Herzberg 2001: 108), voire, ainsi que le peintre le propose lui-même, comme la synthèse de l'ensemble de son parcours de jeunesse: I'art européen du xvI ${ }^{\mathrm{e}}$ siècle, Cézanne, Matisse, la poésie de l'Afrique et la culture cubaine (Leenhardt: 210). Mais elle ouvre aussi un tout nouvel espace. Si l'on peut écrire, à l'instar de Leenhardt, que «ce sont Les Baigneuses de Cézanne et Les Demoiselles d'Avignon de Picasso, dans un décor caraïbe et comme enchanté de divinité afro-cubaine» (ibid.), il faut souligner que ces figures qui évoquent le souvenir du cubisme, ces masques africains «ressurgis [...] comme une réminiscence ancestrale $\mathbf{8}_{\gg}$ font émerger un lieu singulier qui n'est pas simplement un décor, un arrière-plan, mais un type particulier d'espace: un champ de cannes à sucre. Pour le dire autrement, La Jungle renverse les principes classiques de représentation par les moyens modernistes que l'on sait, mais ici, ceux-ci sont portés à un autre niveau, comme transfigurés par la spatialité de ce champ de cannes qu'ils découvrent. Les questions esthétiques jusqu'ici liées au dépassement de la logique figurative, au travail intérieur du peintre, sont déplacées dans cet autre espace, elles y prennent un autre sens. Essayons de préciser en quoi cela consiste.
7. Les dates varient en même temps que les circonstances dans lesquelles Lam aurait eu accès aux statues et masques africains. Pierre Mabille et Fernando Ortiz évoquent la date de 1928 à Madrid; mais si tel est le cas, rien ne transparaît dans la peinture de Lam à l'époque. D'autres critiques pensent qu'il est plus probable que Lam, comme il le raconte lui-même, y ait été confronté pour la première fois en 1938 chez Picasso. Voir: Paudrat 2001: 74 .

8. Cette déclaration est rapportée dans Rousseau 1948 : 591. 
Études et essais

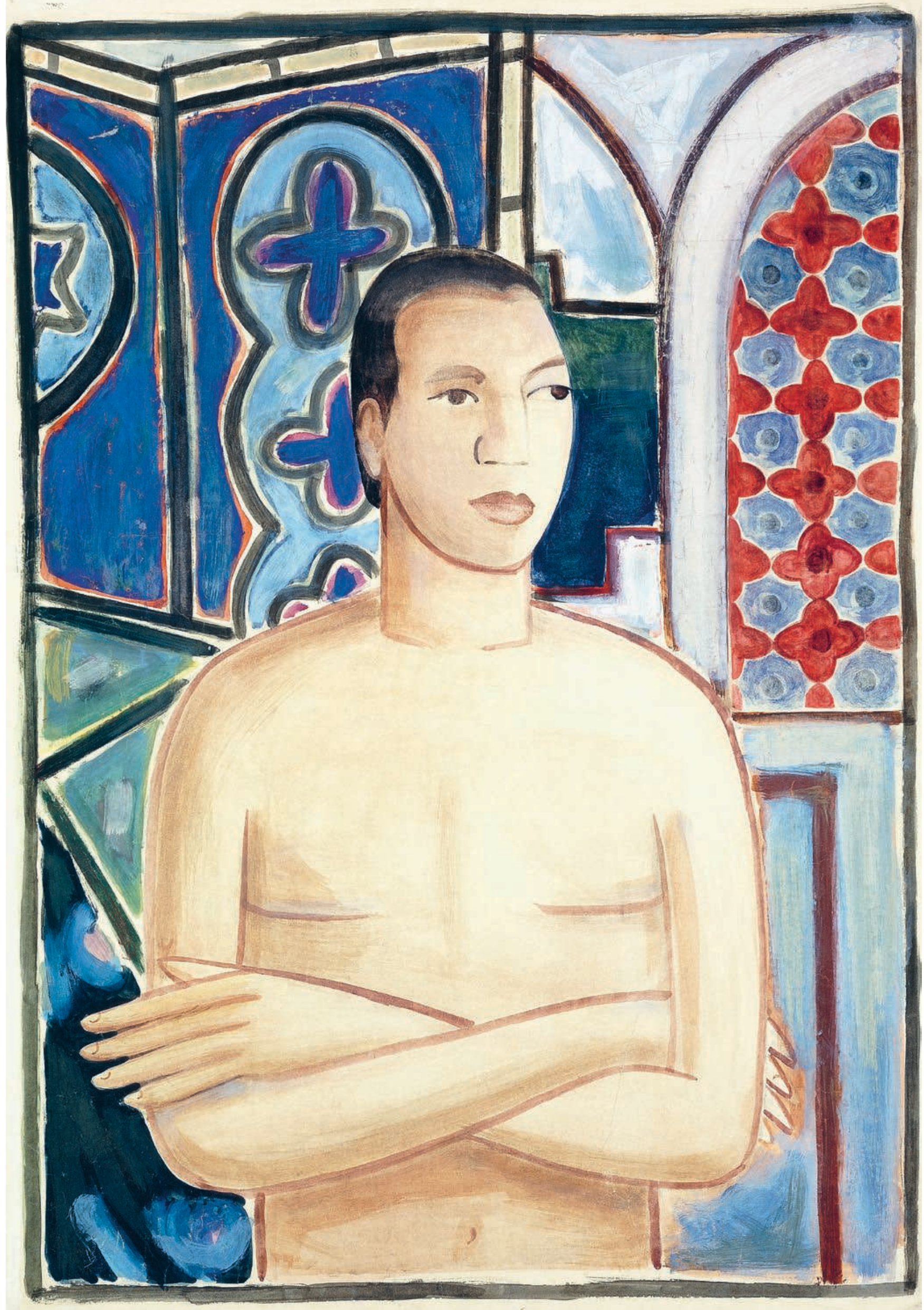

Wifredo Lam, Autoportrait, vers 1938. Gouache et aquarelle sur papier contrecollé sur toile, $90 \times 63,5 \mathrm{~cm}$. Photo ๑ Christie's Images / Bridgeman Images @ Paris, ADAGP, 2021. 
Pierre Mabille est l'un des commentateurs qui ont le plus insisté sur la rupture que La Jungle consommait avec les lois de la perspective, qu'il considérait comme le socle d'une civilisation occidentale en décomposition. Il avait vu La Jungle en 1943 et déclarait:

\section{À tort ou à raison, je considère ce tableau comme un évènement d'importance com- parable aux découvertes de Paolo Uccello surla perspective [...], d'une importance telle qu'elles marquèrent toute la sensi- bilité occidentale [...] [où] ce sont tou- jours les mêmes lois de la perspective qui interviennent. D'autres lois régissent la composition de la Jungle. \\ (Mabille: 119-1209)}

Mais quelles sont ces autres lois? Faut-il simplement les rapporter à l'antiperspective du cubisme ou de l'expressionnisme, à ces lois de l'espace psychophysiologique que bafoue l'ordre de la perspective? Mabille semble vouloir suggérer plus, mais ne va pas véritablement plus loin. Quelques décennies plus tard, Édouard Glissant reprend cette critique à son compte, pour proposer l'idée forte selon laquelle l'espace de La Jungle est inédit parce que, en remettant en cause ces principes de représentation, il ouvre une spatialité immédiatement chargée d'un sens historique: le champ de cannes tel qu'il est peint par Lam retrouve dans son organisation spatiale elle-même sa signification historique, celle d'être le terrain de l'exploitation coloniale (Glissant 2001:13-48). En homme des Antilles, Glissant découvre ainsi cette connaissance historique immanente à la composition de La Jungle: les principes qui la régissent, dit-il, reposent sur les lois qui ont gouverné la coupe de la canne à sucre, c'est-à-dire qu'à ses yeux le tableau est peint et doit être regardé selon le rythme de travail observé par les esclaves, selon la délimitation physique entre le travail à faire et à accomplir: «frontière entre les malheurs qui sont accomplis [...] et cette souffrance encore à venir que cette limite d'une coupe dans un champ devant laquelle s'acharnent les coupeurs et les amarreuses» (ibid.: 15). La lecture de Glissant est capitale dans la mesure où elle vient non seulement compléter celle de Mabille, en donnant au bouleversement spatial une dimension historique précise et un sens à ces lois alternatives de l'espace, mais il redéfinit également l'expérience, le vécu qui se trouve en puissance dans le tableau:

Vous y repérerez certes la nervure des bouts de cannes [...] mais vous y ressentirez surtout l'évidence de cette limite de la coupe, de cette frontière mouvante, non pas entre une proximité et une profondeur, mais entre deux états de l'étendue, également proches et lointains: ce qui est accompli [...] et ce qui reste à démêler dans la souffrance.

(Ibid.)
C'est précisément à partir de cette lecture qu'il nous semble possible de réfléchir au lien entre l'œuvre de Lam et sa part chinoise que nous avons appelée «historique». À Cuba comme dans toute la Caraïbe, la plantation de cannes à sucre est chargée immédiatement du lourd symbole de l'exploitation coloniale. Mais l'abolition de l'esclavage n'y datant que de 1886, «Cuba fut unique au sens où sa florissante industrie sucrière employa les Chinois engagés par contrat synallagmatique, non pas après la fin de l'esclavage des Africains, mais en même temps. De nombreuses plantations cubaines fonctionnaient simultanément avec des esclaves et des contractuels» (Lai 2009: 34)10. Ainsi, ce tableau qui cherche à peindre «l'esprit des Noirs» de Cuba, selon Lam (Fouchet 1998 [1976]: 203), touche aussi - peut-être inconsciemment (mais n'est-ce pas là l'automatisme qui parle?) - les autres expériences liées à cet espace d'exploitation, celui-là même auquel est aussi rattachée l'histoire de la «migration des coolies » chinois. II se charge en outre d'une forte signification biographique dans la mesure où Lam Yam, qui fit le long voyage, de Canton à Cuba, en passant par San Francisco puis Mexico, fit partie de ceux qui formèrent la colonia china, première communauté chinoise de Cuba, et connurent le labeur du champ de cannes - car ce n'est que plus tard, semble-t-il, que le Chino Enrique (nom chrétien qui tend à indiquer l'appartenance à une habitation) se fit commerçant et écrivain public dans le quartier chinois où le peintre vécut jusqu'à l'âge de 12 ans 11 .

Mais si Lam rejoint donc cette part chinoise historique et familiale, c'est bien à travers son œuvre qu'il le fait, c'est par cette spatialité particulière à $L a$ Jungle. S'il rejoint cette expérience historique, ce n'est nullement par la description réaliste des «hommes du sucre» au travail. Glissant l'a montré, il faut approcher La Jungle dans le savoir du labeur de la coupe, mais ce n'est nullement une «remise de paysage» selon son expression. On ne peut pas dire en effet: il y a le champ et dans ce champ il y a des personnages; non, ces personnages sortis des tréfonds de Lam lui-même procèdent de ce milieu, ils n'en sont pas séparés, on pourrait les appeler des «hommes-cannes»: I'un est peut-être un coupeur des champs qui est aussi une prostituée des bas-fonds qui est elle-même une tige de canne à sucre; l'autre une amarreuse, grandie parmi les feuilles, et dont le bras-branche est celui d'un cimarron vengeur. Lam ne trouve-t-il pas ici ces lois qui commandent à ce qui est au-delà de tout réalisme? Surréalisme qui s'affirme d'emblée dans la façon dont se présente l'image du champ, dont les tiges, les feuilles sont mêlées à d'étranges figures qui ne se distinguent pas de cette végétation, mais où, en même temps, l'abrutissement et l'épuisement, la prostitution et le meurtre sont aussi la «jungle» du champ, c'est-à-dire sa profondeur essentielle en tant qu'espace de l'exploitation de l'homme par I'homme. Ainsi chez Lam, la démarche surréaliste qui est de se porter vers l'ombre, d'abandonner le contrôle à l'intériorité inconsciente, se dote immédiatement de cette signification historique - et ce n'est que par là que cette part chinoise historique est rejointe.
9. Dans un autre article, «La peinture rituelle de Wifredo Lam », Mabille reprend une idée similaire où il considère la perspective comme la caractéristique de la culture occidentale traditionnelle, traduite en peinture par les principes développés par les maîtres italiens de la Renaissance et qui ont déterminé la représentation du réel (Mabille 1989 [1938-1951] : 127).

10. Pour une étude détaillée de ces conditions historiques et de la naissance de cette communauté de coolies chinois côtoyant les esclaves, mais aussi les travailleurs indiens, voir: López 2013: 15-53. Entre 1860 et 1870, Sagua la Grande, lieu important de production de sucre, et future ville natale de Lam, fit venir de nombreux contingents d'esclaves africains et de Chinois sous contrat (ibid. : 28).

11. Les dates de Lam Yam ne sont pas certaines: Max-Pol Fouchet, le biographe du peintre, parle d'une mort à l'âge de 108 ans en 1926. Pierre Mabille, autre proche de Lam, donne 1928 comme date de décès, à 103 ans. Les dates

approximatives seraient: 1818-1926 ou 1825-1928. Son arrivée à Cuba se situe selon toute vraisemblance entre 1847 et 1874, c'est-à-dire entre l'arrivée au port de Régla de l'Oquendo, premier bateau chargé de deux cents travailleurs chinois, qui ouvrit la période des «contrats synallagmatiques» pour les coolies, jusqu'au moment où le Rapport de la Commission de Cuba y mit un terme. Dans notre introduction, la citation de Lam qui évoque «le drame de la mer de Chine» n'est pas très clair, mais on peut penser qu'il fait référence au périple entrepris par son père, ou peut-être plus largement aux conditions géopolitiques incertaines. Pour ces données biographiques sur Lam Yam, voir notamment: Fouchet 1998 [1976]; Hu-Dehardt 2013: 98-99. 


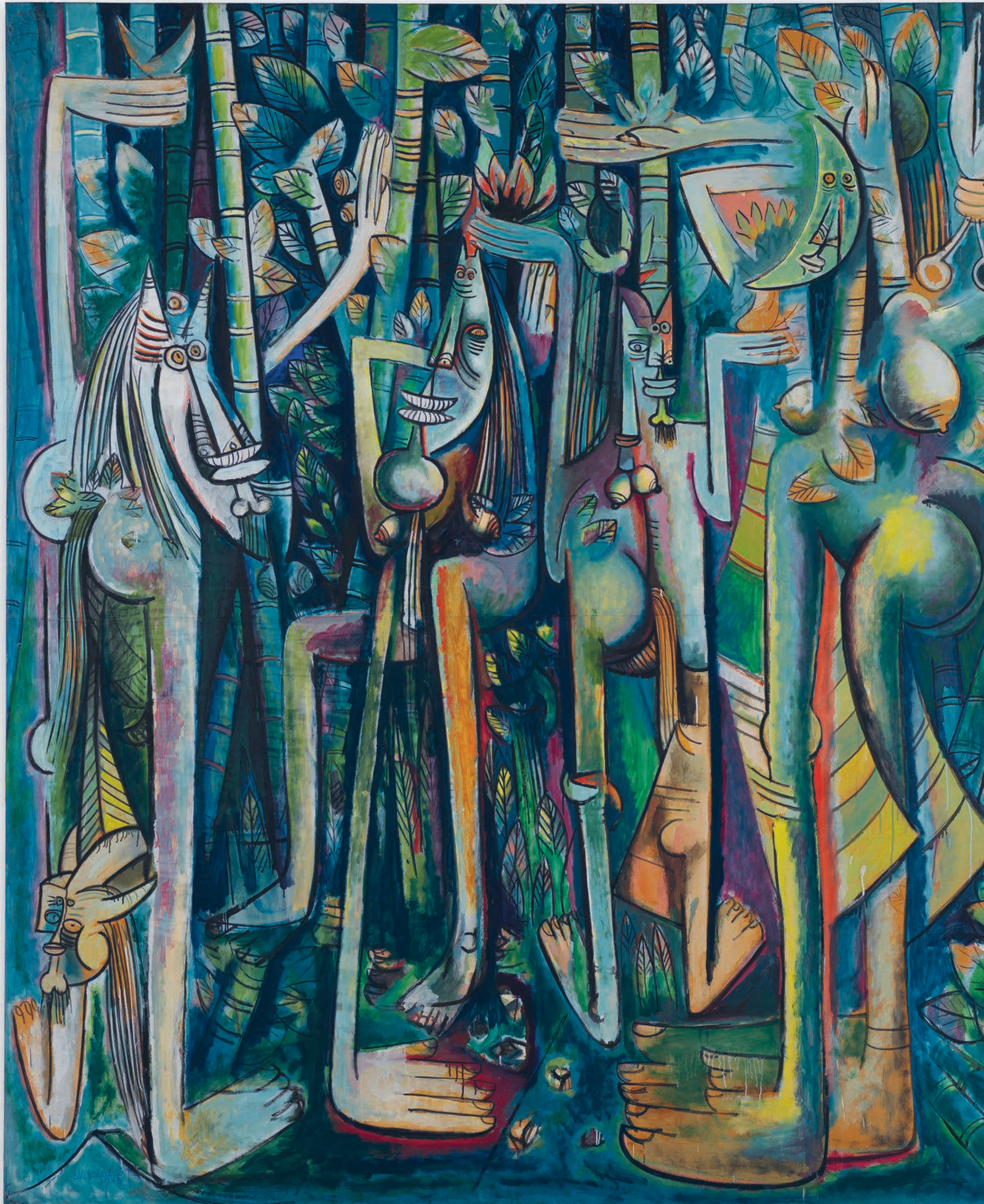


Wifredo Lam, La Jungle, 1942-1943. Huile sur papier marouflé, 239,4 × 229,9 cm. Image digitale, The Museum of Modern Art, New York/Scala, Florence ๑ Paris, ADAGP, 2021 
12. Xuriguera et d'autres comme lui voient dans La Jungle une végétation de bambous.
Mais on perçoit qu'il y a là comme une réponse, mieux, un accomplissement de l'expérience tant désirée par Chen, qui cherchait un modèle intérieur alternatif aux lois de la ressemblance formelle. C'est-à-dire que l'expérience de Lam, en rejoignant la part chinoise historique, serait aussi l'aboutissement de l'idéal de la peinture lettrée au moment de la modernité. On jugera peut-être audacieuse une pareille idée, essayons de l'expliquer. Dans l'argumentaire de Chen, celui-ci s'abritait sous l'autorité de Su Shi. Or derrière la citation qu'il faisait en forme de camouflet, se tient l'imposante réflexion du lettré de la dynastie des Song sur l'expérience de I'homme accompli, l'homme de lettres au sens large et profond de wen 文, dont le destin devait être de s'accorder à la «marche» du monde - cela pourrait traduire l'idée que recouvre le dao 道 - jusqu'à s'y perdre soi-même; et pour s'y perdre, il lui fallait accéder en chaque chose à l'agencement interne, $l i$ 理, et non s'arrêter à leur apparence, toujours fallacieuse au regard du principe qui engendre la multitude des transformations. En peinture, cette pensée s'exprime dans les deux citations suivantes, fort connues, qui se répondent - et qu'il nous faut lire en gardant à l'esprit que c'est Lam, le peintre de l'image automatique du champ de cannes, qui est pour nous le pendant moderniste de l'expérience ici décrite:

Or les peintres d'aujourd'hui peignent les bambous en ajoutant nœud après nœud, feuille après feuille. Cela est contraire au principe interne du bambou, car avant de peindre un bambou, il faut que celui-ci ait déjà poussé en votre for intérieur. C'estalors que, pinceau en main et regard concentré, la chose que vous désiriez peindre se présente: poursuivez-la prestement de votre pinceau, un instant d'hésitation et la vision s'évanouit. Tel estl'enseignement de Yuke. Toutefois, j'ai beau en connaître le principe, par manque de pratique je n'atteins pas la fluidité, l'intérieur et l'extérieur ne fusionnent pas, le cœur et la main ne vont pas l'amble.

(Cheng 2006: 85, traduction modifiée)

Lorsque Yü-k'o peignait un bambou, il voyait le bambou et ne se voyait plus. C'est peu dire qu'il ne se voyait plus:

Dans l'extase, il abandonnait son corps et son corps devenait bambou, faisant jaillir sans fin de nouvelles fraîcheurs [...]. (Ibid.: 86, traduction modifiée)

Lues dans ce contexte, ces descriptions par Su Shi de la peinture de bambou résonnent fortement avec le rapport que le peintre Lam entretient vis-à-vis de la canne à sucre. Bien sûr on pourrait, si ce n'était une facile analogie, faire de Lam un peintre de bambous à proprement parler, tant la tige de la canne, les nœuds qui en marquent les sections, sa verdeur, sa gracile verticalité ressemblent à s'y méprendre aux attributs du «gentilhomme du froid $\mathbf{1 2}$ ». Néanmoins cela est de peu d'importance, on ne doit justement pas s'arrêter à l'apparence formelle - même si elle implique déjà dans ce cas-là une métamorphose remarquable -, il faut plutôt tenter de comprendre comment la maturation intérieure, obéissant à ce grand principe qu'est «l'agencement interne des choses» (Ii), et l'espèce d'extase qui en résulte peuvent être actualisées dans l'expérience de Lam: comment, à travers sa pratique, la chose, qu'elle soit canne ou bambou, s'est développée à l'intérieur de lui-même selon son principe, pour que, ensuite, cette intériorisation se déploie au-dehors et prenne la forme d'une image - image au-delà de toute ressemblance formelle? L'intériorisation de la canne qui, comme le bambou de Yuke, mûrit en son for intérieur a été rendue possible par le lent travail sur l'intériorité créatrice, débuté depuis l'Espagne et qui, au moment du retour à Cuba, notamment grâce à la pratique de l'automatisme, retrouve cette forme au fond de soi. Lam en livre une image qui rejette la puérile ressemblance formelle, ne reproduit pas «nœud après nœud, feuille après feuille» les cannes à sucre des plantations de Cuba, mais en découvre comme le «principe interne». Celui-ci a cependant changé. Le lettré l'assimilait à un "savoir-faire" dicté par les choses elles-mêmes au corps et à la puissance spirituelle (shen 神)» qui permettait la fusion avec l'Origine (Cheng 1997: 443). Ici, les leçons de l'art africain, la spontanéité automatique évoquent un même abandon à la dictée des choses elles-mêmes et, par cet abandon, la libération vis-à-vis des lois objectives de la ressemblance formelle. Elle prend en outre une signification historique bien différente, car le savoir inhérent à la canne à sucre saisie par le pinceau de Lam n'ouvre pas à la connaissance du Ciel-Terre mais à cette connaissance de l'espace de l'exploitation coloniale. La Jungle serait alors l'œuvre d'une communication entre une expérience intérieure semblable à celle du lettré et l'environnement colonial dont elle retrouve les lieux chargés de toute une mémoire historique, ancienne et récente. Elle aboutit, elle aussi, à une sorte de fusion, I'homme n'est plus distinct de son milieu, il ne s'y identifie pas mais il s'y perd, comme si lui-même était devenu partie de la végétation, un «homme-canne». Tel serait le sens, selon nous, de cette double part chinoise dont Lam, au carrefour des métissages, est porteur: le peintre qui a vécu cette «poussée en lui-même», pour parler comme Su Shi, de la canne à sucre livre une œuvre où la dimension esthétique, dont se réclame la peinture lettrée «avancée», se fond dans celle, historique, des travailleurs de la canamelle, esclaves comme coolies. Dans l'exacte mesure où il accède à l'expérience du peintre lettré, Lam affirme sa créolité, et comme Yuke, sous le Ciel, devenaitl'hommebambou, Lam devient l'homme-canne d'une modernité déracinée.

tǵbre@yahoo.com 
La part chinoise de Wifredo Lam, perspectives historiques et esthétiques Pierre-Mong Lim

\section{BIBLIOGRAPHIE}

Andrews, Julia et Shen, Kuiyi

2006 «The Japanese Impact on the Republican Art World: The Construction of Chinese Art as a Modern Field», TwentiethCentury China 32: 4-35.

Breton, André

1965 [1928] Le Surréalisme et la peinture. Paris, Gallimard.

Chen, Duxiu 陳獨秀

1998 《Meishu geming» 美术革命 (La révolution en art), in Gu Sen 顾森, Bainian Zhongguo meishu jingdian wenku Zhongguo chuantong meishu 百年中国美术经 典文库. 中国传统美术

(Anthologie de textes sur l'art chinois du centenaire, l'art

traditionnel chinois)

Pékin, Haitian chubanshe.

Chen, Shizeng 陳師曾

2010 Zhongguo huihuashi中國繪畫 史 (Histoire de la peinture chinoise) Pékin, Zhonghua shuju.

Cheng, François

2006 [1989] Souffle-Esprit: textes théoriques chinois sur l'art pictural. Paris, Seuil.

\section{Chow, Tse-tsung}

1967 May Fourth Movement: Intellectual Revolution in Modern China. Stanford, Stanford University Press.

\section{David, Catherine}

2015 Wifredo Lam, catalogue d'exposition (Paris, Musée national d'art moderne-Centre de création industrielle, sept. 2015-fév. 2016). Paris, Centre Pompidou.

Fouchet, Max-Pol

1998 [1976] Wifredo Lam. Paris, Cercle d'Art.

\section{Glissant, Édouard}

2001 «lguane, busard, totems fous: I'art primordial de Wifredo Lam», in Christiane FalgayrettesLeveau et al., Lam: métis, catalogue d'exposition (Paris, musée Dapper sept. 2001-janv.2002): 13-48. Paris, Dapper.

\section{Herzberǵ, Julia}

2001 «Naissance d'un style et d'une vision du monde: le séjou à la Havane, 1941-1952», ibid.

\section{Hu-Dehardt, Evelyn}

2013 «Integration and Exclusion: The Chinese in Multiracial Latin America and the Caribbean»", in Tan Chee-Beng (dir.), Routledge Handbook of Chinese Diaspora. New York, Routledge: 89-107.

Kahnweiler, Daniel-Henry

1948 "L'art nègre et le cubisme», Présence africaine 3: 367-377.

Kang, Youwei 康有為

1998 Wanmucaotang canghua mu $x u$ 萬木草堂藏畫目序 (Collection de peintures du Pavillon luxuriant) [1918], in Gu Sen 顾森, Bainian Zhongguo meishu jingdian wenku Zhongguo chuantong meishu
百年中国美术经典文库·中国传统美 术 (Anthologie de textes sur l'art chinois du centenaire, l'art traditionne chinois). Pékin, Haitian chubanshe.

Lai, Walton Look

2009 «Asian Diasporas and Tropical Migration in the Age of Empire: A Comparative Overview $»$, Journal of Chinese Overseas 5: 28-54.

Laude, Jean

1972 «Picasso et Braque, 1910-1914 la transformation des signes», in Le Cubisme, actes du $1^{\text {er }}$ colloque d'histoire de l'art contemporain (musée d'Art et d'Industrie Saint-Étienne, nov. 1971): 7-28. Saint-Étienne, Cierec.

2006 [1968] La Peinture française et l'art nègre. Paris, Klincksieck.

Leenhardt, Jacques

2009 Lam. Paris, HC Éditions.

Leiris, Michel

2011 Écrits sur l'art, Pierre Vilar (éd.). Paris, CNRS Éditions.

López, Kathleen

2013 Chinese Cubans: A Transnational History. Chapel Hill, UNC Press.

Mabille, Pierre

1989 [1938-1951] Conscience lumineuse, conscience picturale, Jacqueline Chénieux-Gendron et Rémy Laville (éd.). Paris, José Corti.

\section{Murata, Hiroshi}

2005 «Wifredo Lam Reconsidered: Modernism and Beyond», Bulletin of Atomi Junior College 42: 68-91.

Núñez Jiménez, Antonio 1982 Wifredo Lam. La Havane, Letras Cubanas.

Pang, Shiwei 庞世伟

$\mathbf{2 0 0 7}$ «Wenrenhua zhi jiazhi de wenti bianlun ji qi yinxiang» 论五四时期「文人画之价值」的问题 辩论及其影响 (Le débat autour de la question de «la valeur de la peinture lettrée» au moment du 4-Mai et ses répercussions), Mei yu shidai 11: 42-45.

\section{Pann, Lynn (dir.)}

2000 Encyclopédie de la diaspora chinoise, trad. de l'anglais par

Thierry Piélat et al. Paris, Éditions du Pacifique.

\section{Panofsky, Erwin}

1975 La Perspective comme forme symbolique, trad. de l'allemand par Guy Ballangé et al. Paris, Minuit.

\section{Paudrat, Jean-Louis}

2001 «Afrique en confluence», in Christiane Falgayrettes-Leveau et al., Lam: métis, catalogue d'exposition (Paris, musée Dapper, sept. 2001-janv.2002): 73-100. Paris, Dapper.

Rousseau, Madeleine

1948 «Un peintre cubain», Présence Africaine 4: 590-594.
Schwarez, Vera

1986 The Chinese Enlightenment: Intellectuals and the Legacy of the May Fourth Movement of 1919. Berkeley, University of California Press.

Schwartz, Benjamin

1972 Reflections on the May Fourth Movement: A Symposium. Cambridge, Harvard University Press.

\section{Sims, Lowery Stokes}

2002 Wifredo Lam and the International Avant-Garde, 1923-1982. Austin, University of Texas Press.

Su, Dongpo 蘇東坡 2008 [1957] «Wen Yuke hua Yundanggu yanzhu ji» 文與可畫筫䈏谷儤竹記

(Notes sur Yuke peignant des bambous dans la vallée des hauts bambous), in Yu Jianhua 俞剑华, Zhongguo gudai hualun leibian 中国古代画论类编 (Anthologie des traités de peinture de la Chine ancienne). Pékin, Renmin meishu chubanshe.

2008 [1957] «Shu Chao Buzhi suocang Yuke hua zhu san shou» 書昆補之所藏與可畫竹三首 (Poème en trois strophes sur un rouleau peint par Yuke, détenu par Chao Buzhi), in Yu Jianhua 俞剑华, Zhongguo gudai hualun leibian 中国古代画论类编

(Anthologie des traités de peinture de la Chine ancienne). Pékin Renmin meishu chubanshe.

Su, Shi

2010 Commémorations, trad. du chinois par Stéphane Feuillas. Paris, Les Belles Lettres.

Tronche, Anne

2010 «Une morphologie totémique de l'invisible», in Blandine

Chavanne et al., Wifredo Lam: voyages entre Caraïbes et avantgardes, catalogue d'exposition (musée des Beaux-Arts de Nantes, avr.-août 2010): 19-43. Lyon, Fage éditions.

\section{Wang, Gungiwu}

1992 [1981] Community and Nation: China, Southeast Asia and Australia. Sidney, Allen \& Unwin.

\section{Xuriguera, Gérard}

1974 Wifredo Lam. Paris, Filipacchi.

\section{Zhuang, Guotu}

2013 «Chinese Policies on Chinese Overseas», in Tan Chee-Beng (dir.), Routledge Handbook of Chinese Diaspora. New York, Routledge:

31-35. 